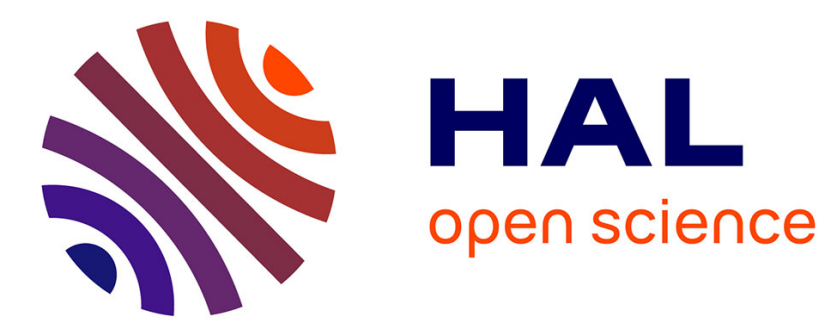

\title{
Jouer de l'ordinateur sur un air urbain
}

Denise Pumain, Thérèse Saint-Julien, Michel Vigouroux

\section{To cite this version:}

Denise Pumain, Thérèse Saint-Julien, Michel Vigouroux. Jouer de l'ordinateur sur un air urbain. Annales de géographie, 1983, 92 (511), pp.331 - 346. 10.3406/geo.1983.20191 . halshs-01488298

\section{HAL Id: halshs-01488298 https://shs.hal.science/halshs-01488298}

Submitted on 13 Mar 2017

HAL is a multi-disciplinary open access archive for the deposit and dissemination of scientific research documents, whether they are published or not. The documents may come from teaching and research institutions in France or abroad, or from public or private research centers.
L'archive ouverte pluridisciplinaire HAL, est destinée au dépôt et à la diffusion de documents scientifiques de niveau recherche, publiés ou non, émanant des établissements d'enseignement et de recherche français ou étrangers, des laboratoires publics ou privés. 


\section{Jouer de l'ordinateur sur un air urbain}

Denise Pumain, Thérèse Saint-Julien, M. Vigouroux

\section{Citer ce document / Cite this document :}

Pumain Denise, Saint-Julien Thérèse, Vigouroux M. Jouer de l'ordinateur sur un air urbain . In: Annales de Géographie, t. 92, n`511, 1983. pp. 331-346;

doi : 10.3406/geo.1983.20191

http://www.persee.fr/doc/geo_0003-4010_1983_num_92_511_20191

Document généré le 15/06/2016 


\title{
Résumé
}

Comment et pourquoi l'analyse quantitative s'est progressivement imposée auprès d'une minorité importante de chercheurs français en géographie urbaine ? L'article répond à ces questions. L'incursion des géographes urbains dans ces nouveaux champs méthodologiques et techniques a beaucoup contribué à la vitalité des recherches urbaines et ce pour plusieurs raisons. La première est que grâce à la généralisation de l'outil informatique et aux possibilités de l'analyse multivariée des vérifications jusque là irréalisables ont pu être faites dans différents domaines, et que par ailleurs de nouveaux champs d'observation ont été ouverts (exemple : la télédétection). La seconde est que les démarches mises ainsi en œuvre ont renvoyé le chercheur à des exigences beaucoup plus grandes en matière d'explicitation théorique, de conceptualisation, et de rigueur de l'observation. Les tenants de cette démarche se trouvent donc très étroitement impliqués dans l'ensemble des débats et confrontations qui animent depuis une dizaine d'années la géographie urbaine française. Aujourd'hui, les voies ouvertes par la modélisation et l'analyse des systèmes apportent de nouvelles perspectives à l'investigation urbaine.

\begin{abstract}
How and why has quantitative analysis imposed itself upon an important minority of French urban geographers ? This article answers these questions. The urban geographers'entry into these new methodological fields and these new techniques has much contributed to the vitality of urban studies for several reasons. First the spreading of computers and the possibilities of multivariate analysis have enabled to make verifications so far impossible to perform in several fields; besides new means of study have opened up (e.g. remote sensing). Second such processes are far more demanding regarding the theoretical explanation, the conceptualization and the precision of observation of the phenomena. The followers of these processes are thus very much involved in the debates that have been at the center of French urban geography for the last ten years or so. To-day model-making and system-analysis are opening new ways and hopes for the urban studies.
\end{abstract}




\title{
Jouer de l'ordinateur \\ sur un air urbain
}

\author{
D. Pumain, \\ Th. Saint-Julien \\ et M. Vigouroux
}

Le grand intérêt que les géographes ont depuis une vingtaine d'années accordé aux recherches urbaines est indissociable de la formidable explosion démographique des villes qui s'est manifestée après la seconde guerre mondiale sur l'ensemble de la planète. La géographie urbaine est sans doute aujourd'hui en France le domaine le plus fréquenté de la discipline. Les champs géographiques sur lesquels ont porté ses recherches se sont considérablement élargis. On peut noter par exemple, l'intérêt croissant porté à l'urbanisation du Tiers Monde, ou encore au développement urbain des pays socialistes. A cet élargissement géographique s'est conjuguée une grande diversification des problématiques et des démarches. Il serait passionnant et fructueux, pour une histoire de la pensée et de la pratique des géographes, d'entreprendre un bilan d'ensemble de cette période récente de la géographie urbaine française, qui aura été, nous le pensons, extrêmement féconde. Notre contribution est plus modeste. Nous cherchons simplement à dire l'histoire et évaluer la portée de la rencontre entre la géographie urbaine et les outils du traitement automatique de l'information. Les contributions à cette rencontre ont été très nombreuses, en témoigne la longue bibliographie qui figure à la fin de cet article. Il est probable que, compte tenu de l'extrême dispersion des publications géographiques, nous ayons commis quelque oubli. Que les auteurs oubliés veuillent 
n'y voir aucune exclusive, le bilan proposé ici n'est qu'une esquisse nécessairement infléchie par notre propre expérience.

Au début des années soixante-dix, les recherches quantitatives et théoriques se sont largement développées dans les différents champs de la géographie urbaine française. Le pas alors franchi était à bien des égards inéluctable. Inéluctable d'abord, parce qu'il était la conséquence logique du renouvellement de la problématique des recherches urbaines intervenu dans la décennie précédente: on n'espérait plus que le progrès des connaissances sourdrait de l'accumulation des monographies de villes: on l'attendait désormais comme le fruit de grandes études comparatives. Le Fait Urbain (Carrière, Pinchemel, 1963), novateur dans son principe, ajoutait à un vaste tableau de l'ensemble des villes françaises le test de certaines hypothèses d'économie urbaine émises par des auteurs anglo-saxons, et pressentait, sans pouvoir les réaliser, la richesse et la puissance des analyses de corrélation. La série des travaux sur l'armature urbaine française (Chabot, 1961; Hautreux, Lecourt, Rochefort, 1963), d'une importance exceptionnelle pour la connaissance du réseau, ne disposait pas des outils d'analyse adaptés à la masse et à la nature des données regroupées. Un dernier exemple enfin, celui de l'atlas de Paris et de la région parisienne (BeaujeuGarnier, 1967). De l'aveu même de son principal maître d'œuvre (1971), il ne pouvait être que la dernière entreprise de cette envergure à ne pas bénéficier, durant les étapes successives de sa réalisation (saisie des données, analyse statistique, réalisation cartographique), des capacités multiples de l'outil informatique.

Ces grandes études comparatives (les trois exemples évoqués sont très significatifs) ont posé aux chercheurs en géographie urbaine des problèmes méthodologiques nouveaux dont la solution ne pouvait être trouvée dans l'expérience passée. Ce sont donc, initialement, des questions méthodologiques et techniques qui ont été les plus mobilisatrices, sans rupture apparente avec les composantes les plus vivantes de la recherche urbaine française. Cette absence de discontinuité a été favorisée par le fait qu'entre 1960 et 1970, les géographes urbains français ont activement participé aux études préparatoires aux plans et programmes d'aménagement du territoire, alors élaborés à différentes échelles. Cette participation les a mis en contact avec d'autres disciplines (économie, sociologie, statistique) que celles avec lesquelles ils avaient traditionnellement l'habitude de cohabiter, et avec des praticiens de l'espace qu'ils connaissaient jusque-là assez peu (architectes, aménageurs divers). Ils ont été confrontés à une très grande diversité de courants de pensée et à des formes de raisonnement qui leur étaient parfois un peu étrangères. Leur champ de réflexion ne pouvait que sortir enrichi de cette large confrontation pluridisciplinaire. 
C'est dans ce contexte que les travaux de P. Claval (1968) ont grandement contribué à faire connaître aux géographes français les recherches urbaines anglo-saxonnes. Ces recherches qui, depuis une dizaine d'années, s'efforçaient d'expliciter le référentiel théorique de la géographie urbaine, sous l'influence de la démarche expérimentale qu'impose assez rapidement l'analyse quantitative (Berry 1958, King 1961, Berry 1968, Berry, Marble 1968 par exemple), ont naturellement trouvé un écho, à défaut de susciter une adhésion massive et passive.

Toutes ces convergences ont contribué à un profond renouvellement de la géographie urbaine française que la généralisation de l'outil informatique a, par ailleurs, soutenu. Plus que dans d'autres domaines de la géographie peut-être, les amateurs occasionnels du "gadget informatique " ont été peu nombreux et tôt découragés. Au prix d'un travail approfondi, des questions depuis longtemps posées ont pu être résolues grâce aux facilités de calcul apportées par l'ordinateur. L'introduction des techniques et des méthodes nouvelles a rencontré des réticences voire des résistances et a, par là même, favorisé l'ouverture et l'animation de certains débats épistémologiques importants. Ces techniques et ces méthodes ont, à leur tour, suscité l'émergence de nouvelles interrogations et l'exploration de nouvelles démarches. Il y a tout lieu de penser que nous ne sommes, aujourd'hui, qu'aux débuts de ce processus de renouvellement.

\section{L'informatique pour l'analyse des données}

L'analyse comparative, dans le but d'établir des corrélations entre des faits mesurés dans des situations urbaines différentes, a multiplié les opérations de confrontation de chiffres. Il ne s'agit plus d'extraire, à l'appui d'une hypothèse, un exemple jugé significatif ou représentatif, la démarche requiert une couverture spatiale exhaustive à l'échelon géographique considéré. Le souci d'étayer le raisonnement sur une information très vaste et traitée de la manière la plus transparente possible, autant que la mise à la disposition des chercheurs du matériel informatique et des logiciels de traitement de données, explique qu'une importante minorité de géographes ait choisi d'utiliser l'ordinateur. Dans le même temps, le développement de l'urbanisation et des problèmes associés a contribué à enrichir et à diversifier les sources disponibles pour les études urbaines. Les chercheurs ont réalisé des plans d'enquêtes de plus en plus ambitieux et ont donc été confrontés à la difficulté d'interpré- 
ter la masse des réponses obtenues sans le support irremplaçable des techniques statistiques.

En se généralisant, le recours à l'analyse des données est devenu indispensable. Un exemple extrême est celui de l'exploration du contenu particulièrement riche et complexe des images que livre à l'observateur la télédétection. A cet égard, les travaux de A. Chatelain (1981) sur l'agglomération marseillaise ou encore ceux du groupe Dupont (1980) sur l'évolution des modes d'occupation du sol à Montpellier, sont pleins d'enseignements. Récemment, un colloque a confronté les travaux d'une dizaine d'équipes de chercheurs utilisant la télédétection pour l'étude des milieux urbanisés (C.N.R.S., 1982). Dans le même ordre de préoccupations, on a pu, au moyen de l'analyse statistique, progresser dans la reconnaissance des formes urbaines (Lowy, 1975).

L'essentiel des apports de l'analyse des données en géographie urbaine a pourtant, jusqu'ici, répondu à d'autres préoccupations.

\section{L'écologie urbaine}

En référence plus ou moins explicite aux travaux d'écologie urbaine de l'école de Chicago (Grafmeyer, Joseph, 1979), et dans un sens peut-être un peu réducteur par rapport à ces derniers (Brun, 1981), de nombreuses observations tendaient à définir l'existence de relations entre les caractéristiques socio-économiques des populations urbaines et leur situation géographique dans la ville. Rendant possible la prise en compte simultanée d'un très grand nombre de variables, l'analyse multivariée a permis d'identifier ces relations. On a pu, grâce à elle, mesurer le degré de pertinence et surtout d'universalité de modèles spatiaux élaborés d'après l'observation des villes américaines.

C'est d'ailleurs sur les villes américaines que se sont portées les premières observations et réflexions des géographes français.

L'étude de J.-B. Racine sur Montréal (1973) a familiarisé les chercheurs avec les travaux de l'école de Berry en "écologie urbaine factorielle». A. Bailly (1976) a observé l'espace social d'Edmonton. Plus récemment, B. Marchand (1978) s'est non seulement attaché à définir les composantes socio-économiques qui structurent l'espace de Los Angeles, mais il a mis en œuvre des techniques nouvelles d'analyse spatiale (comme le variogramme) et d'analyse des changements intervenus dans cette structuration. Les agglomérations européennes et surtout françaises ont, elles aussi, bénéficié des acquis de ces nouveaux outils d'analyse. Nous citons, à titre d'exemple, les études sur Milan (Dalmasso et al., 1971), sur l'agglomération parisienne (Lebart, Tabard, 1971; Chauviré, Noin, 
1980), l'observation de l'espace social de Marseille (Roncayolo, 1972), d'Amiens et de Châlons-sur-Marne (Debonneuil, Gollac, 1978), ou encore de Rouen (Guermond, 1981), de Nîmes, Montpellier et Perpignan (Vigouroux, Volle, 1982). Jusqu'ici ces travaux ont été conduits en ordre dispersé. Un bilan d'ensemble des caractéristiques intra-urbaines des villes françaises reste à établir.

En fait, probablement en raison d'une facilité plus grande à se procurer des données, les recherches intra-urbaines utilisant l'ordinateur comme outil d'investigation ont contribué à déplacer, sinon la problématique, du moins le champ étudié : de la morphologie des quartiers, de la composition urbaine au sens urbanistique du terme, de l'organisation spatiale des fonctions, marquée par les équipements et les infrastructures, qu'avait développées la géographie "classique", on est passé à des questions de géographie sociale. L'intérêt des chercheurs s'est alors dirigé vers d'autres interrogations, qui les ont souvent détournés de l'ordinateur.

\section{La perception de l'espace urbain}

Ainsi, dans les études intra-urbaines, c'est depuis peu l'approche comportementale qui connaît la plus grande faveur. En réaction contre certaines hypothèses trop réductrices, sous l'influence de la sociologie, de la pyschosociologie et des travaux de géographes anglo-saxons (dits "behavioristes") une importance croissante a été donnée à toute la subjectivité de la décision humaine dans le façonnement et l'appropriation de l'espace de la ville. Les géographes se sont intéressés aux comportements des acteurs dans la ville et aux processus cognitifs qui engendrent ces comportements. En fait, les travaux de A. Bailly (1977) exceptés, ce courant (Bertrand, 1977, Ferras, 1978, Piolle, 1979, Vant, 1981, etc.) s'est peu intéressé à l'analyse statistique et à la modélisation. L'informatique n'a été utilisée que pour les dépouillements d'enquête (Andan, 1982) et secondairement la cartographie. A. Bailly a exploré comment les propriétés topologiques de l'espace urbain peuvent permettre d'exprimer le vécu quotidien de ses habitants à partir d'observations réalisées sur plusieurs villes de l'Est de la France.

\section{Les études de réseau}

C'est sans doute à l'échelle du réseau urbain français que les vérifications les plus systématiques ont été réalisées. Elles répondent à des questions d'ordre général sur les réseaux, ou plus spécifiques de la situation française, que l'absence d'outils interdisait jusque-là de résoudre. On a, par exemple, évalué la nature, l'intensité et la 
forme des relations qui, dans un système de villes, lient la taille des unités et le degré de leur spécialisation fonctionnelle (Pumain, Saint-Julien, 1978). On a montré quelles correspondances existent entre les profils d'emploi de la population active des unités du réseau et ceux des flux migratoires d'entrée et de sortie au cours des périodes intercensitaires récentes (Pumain, 1980). L'analyse multivariée a aussi permis de qualifier et de comparer des structures urbaines définies par un grand nombre de critères (profils d'emplois, profils démographiques (Haumont, Bohain, 1968), ensemble de caractères socio-économiques (Pumain, Saint-Julien, 1978), variables financières (Bouinot, Maarek, 1974), caractéristiques de l'organisation spatiale interne (Thibault, 1975), critères de desserte périphérique (Andan, Boislaroussie, Geiger, 1976). Les grands acquis de l'ensemble de ces travaux, qui se complètent et se renforcent, sont une meilleure connaissance des disparités socio-économiques qui déterminent à un moment donné la configuration du réseau urbain national. Ils sont aussi dans la mise en évidence du sens des transformations de cette configuration (Pumain, Saint-Julien, 1978; Pumain, 1982, Saint-Julien, 1982). On a ainsi pu vérifier la validité de certaines hypothèses cherchant à rendre compte des aspects les plus récents de la division spatiale du travail entre les villes et de leurs effets sur la configuration sociale du réseau urbain national.

La majorité de ces travaux a, en outre, proposé des classifications en groupes de villes homogènes ou hiérarchisées sur des principes clairement explicités et une fois définis, clairement objectivés. Ces classifications, dont la signification est toujours clairement rattachée à une problématique particulière, ont le grand avantage de donner des résultats contrôlables et reproductibles. Que de chemin parcouru, de la typologie très intuitive de A. Chatelain (1946) à celle de $J$. Hautreux et $M$. Rochefort (1964) puis à celle de C. Lacour (1970) qui, grâce à l'analyse des similitudes, a pu classer les villes françaises en tenant compte simultanément de plusieurs caractères qualitatifs!

\section{De l'analyse à la théorie urbaine: vrais et faux débats}

Nul ne saurait aujourd'hui contester le bond qualitatif que le recours à l'analyse multivariée a permis dans la connaissance du phénomène urbain contemporain, toutes choses égales bien sûr quant aux données initiales mises en œuvre. Très vite d'ailleurs, la réflexion sur ces résultats a conduit à d'autres développements. Ce qui était à l'origine simple perfectionnement technique est progres- 
sivement devenu source d'une démarche scientifique car, de son fait, les questions se sont renouvelées et les centres d'intérêt de la recherche déplacés.

\section{Les résultats de l'analyse des données: des artefacts?}

Si les techniques multivariées aident à condenser l'information réunie, la quantité de résultats produite reste considérable. Leur appréhension n'est pas immédiate et la réflexion sur les «êtres mathématiques" identifiés par exemple en écologie urbaine a soulevé maintes discussions: s'agit-il toujours de véritables « dimensions latentes" de la différenciation des quartiers des villes, qu'il serait possible d'associer chacune à un processus spécifique de construction du tissu urbain? (Pruvot, Kleinweber, 1980) Ou bien, ne faut-il attribuer à ces facteurs qu'une valeur descriptive, quand on ne les considère pas comme autant d'artefacts produits par la méthode? Il y a tout lieu de penser que les travaux attendus de M. Pruvot d'une part, et de $M$. Vigouroux d'autre part répondront au moins partiellement à ces questions. Celles-ci ont été plus précisément posées en écologie factorielle (Brun, 1981) mais elles sont sous-jacentes au traitement de toute matrice d'information spatiale, et les travaux réalisés à l'échelle interurbaine les ont également discutées. Elles renvoient aussi au large débat théorique qui traverse la recherche urbaine française depuis 1968.

\section{Utiliser l'ordinateur = autonomiser l'espace?}

En effet, un débat que nous considérons comme partiellement faux a beaucoup troublé la conscience "de classe » des géographes urbains. Les démarches marxiste d'une part et comportementale d'autre part, pour des raisons peut-être différentes, mais jamais très claires, ont très tôt jeté l'anathème sur l'usage de tous les outils méthodologiques et techniques d'analyse que, progressivement, l'usage de l'ordinateur a généralisés. Les travaux d'H. Lefebvre (1968) et plus précisément ceux de M. Castells (1972) d'une part, et ceux d'A. Lipietz (1977) d'autre part, ont largement étayé depuis une dizaine d'années la réflexion marxiste en géographie sur la ville et l'espace urbain, proposant, à bien des égards, un renversement des problématiques. La ville est étudiée comme une expression parmi d'autres des contradictions du système capitaliste. "La structure de l'espace n'est que la dimension matérielle des rapports sociaux." Les travaux menés par M. Castells et M. Godard (1974) sur les luttes urbaines à Dunkerque ont, pendant quelques années, représenté le symbole des préoccupations nouvelles qui ont animé 
de nombreuses recherches en géographie. Pour des raisons rarement explicitées, les géographes qui ont orienté dans ce sens leurs travaux sur la ville, et qui ont, bien entendu, été confrontés à la constitution et au traitement de bases de données (données originales ou données statistiques fournies par différents organismes) ont, dans l'ensemble, boudé toutes les techniques et les méthodes usuelles indispensables au traitement correct des tableaux de chiffres. Au nom du danger "d'autonomiser l'espace" ou d'une sophistication idéologiquement suspecte des méthodes statistiques proposées (leur utilité est, il est vrai, beaucoup plus communément admise dans les autres disciplines et dans les géographies étrangères que dans la géographie française), ils ont contribué à pérenniser les outils les plus frustes d'investigation. Ainsi, par exemple, le graphique triangulaire, signe de la "belle époque» de l'analyse statistique en géographie, est encore aujourd'hui préféré à tout algorithme de classification pour établir une typologie des profils sociaux d'un ensemble d'unités géographiques (Vant, 1981, pp. 467477). La fiabilité des procédures de classification automatique, la finesse de leurs résultats, le choix qu'elles permettent entre des critères d'agrégation très divers et toujours parfaitement contrôlables ne sont pourtant plus à démontrer.

Pour des raisons tout aussi peu explicites, et sans doute en rapport cette fois avec la déshumanisation du contact du chercheur avec son tableau des données, les travaux de géographie comportementale sont, en France, à peu d'exceptions près (C. Cauvin et A. Bailly par exemple), restés assez peu réceptifs aux aides méthodologiques et techniques mises à leur disposition par l'analyse quantitative. On s'en étonne d'autant plus que ces travaux reposent, en général, sur la réalisation d'enquêtes. Il existe aujourd'hui des techniques dont l'utilité n'est plus discutée pour la réalisation correcte d'un sondage, l'établissement d'un plan d'enquête, l'exploitation des bases de données qui en découlent. Comment faire l'économic de leur usage, puisqu'ils sont là, précisément, pour accroître la fiabilité des résultats finalement proposés?

Il serait dommage qu'un tel faux débat stérilise pour longtemps une part de la géographie urbaine française, en coupant les communications entre au moins trois de ses courants les plus vigoureux. Le refus de l'approche quantitative, sur un plan théorique, au profit d'une approche "marxisante", ou "behavioriste", autorise-t-il un piétinement méthodologique? 
Vers une redéfinition des objets de la géographie urbaine

C'est à partir de questions plus spécifiquement géographiques que d'autres chercheurs ont remis en cause la pertinence de la matrice d'information spatiale, ou encore de la notion de systèmes de villes, formalisées à l'origine par B.J.L. Berry (1964) pour définir les objets de la recherche urbaine.

En effet, dans une perspective systémiste, les unités territoriales ainsi observées ne sont pas, a priori, d'authentiques objets géographiques. Dans le domaine intra-urbain, M. Vigouroux (1981) a fait différentes propositions pratiques pour rapprocher la matrice d'information spatiale du projet qui lui est assigné. On doit, par ailleurs, beaucoup en France aux travaux d'H. Reymond (1980) qui s'est interrogé sur l'identité des objets géographiques pertinents pour définir un système de peuplement en général et un système urbain en particulier. Selon lui, les agrégats habituellement observés (unités urbaines décrites par des caractéristiques très diverses visant à tenir compte du plus grand nombre possible d'attributs) ne sont peut-être pas les unités de base les plus adéquates pour restituer dans le système les véritables propriétés de l'étendue géographique: si les "systèmes d'habitat" ont une relative autonomie, toutes les autres activités urbaines sont trop sous la dépendance de processus externes pour permettre d'identifier la ville dans sa totalité à un système. Cette approche invite à un approfondissement pour une théorie géographique de la ville: "nos systèmes d'habitat urbain apparaissent comme les organismes écologiques de base de l'espèce humaine... apprécier les équilibres locaux entre charge humaine et sites bio-géographiques s'impose comme une tâche primordiale et permet de préciser le contenu souhaitable de la géographic urbaine. Si l'on admet qu'un réseau urbain n'est, dans ses formes actuelles les plus courantes, pas autre chose qu'un système d'articulation spatiale de sites de ressources - renouvelables et non renouvelables - grâce à l'interconnexion des situations de richesse - que représente chaque agglomération - par les transports physiques de biens et de personnes, alors le rôle dévolu à une géographie urbaine "informatisée " s'éclaire... Les interactions à prendre en compte sont, on le voit, extrêmement nombreuses: économiques, écologiques, sociales, géographiques; elles exigent qu'on n'utilise plus seule la vieille causalité linéaire et qu'on aborde avec rigueur la masse des données à traiter» (H. Reymond, 1980, pp. 10-12). 


\section{De la théorie à la modélisation: retour à l'informatique}

Grâce à l'utilisation des capacités de calcul de l'ordinateur et des techniques d'analyse de données, les études empiriques réalisées de manière systématique et comparative ont permis d'établir un certain nombre de régularités, de tester un grand nombre d'hypothèses, entraînant l'approfondissement et le renouvellement de la réflexion théorique. La démarche scientifique tient pour garant de la validité de celle-ci son aptitude à la prévision de résultats ultérieurement confirmés par l'expérience. Compte tenu de la complexité et de l'historicité des situations dans les sciences humaines, on substitue à cette démarche expérimentale la modélisation et la simulation - c'est alors que l'ordinateur redevient un outil essentiel pour l'avancement de la recherche.

Cette étape n'est apparue que tardivement dans la pratique des géographes. La recherche urbaine sur modèles est longtemps restée l'apanage des bureaux d'études (S.E.M.A., B.E.T.U.R.E., I.A.U.R.I.F...), des économistes ou des ingénieurs. Leur apport n'est alors pas toujours limité à la recherche appliquée: les travaux de R. Bussière (rassemblés dans son dernier ouvrage: 1982), sur la distribution des densités urbaines et des tailles de villes, sont une contribution très importante à la modélisation de la géographie du peuplement, à l'échelon intra et interurbain.

Les travaux des géographes français ont d'abord consisté à tester des modèles existants et à en proposer des améliorations pour les adapter aux observations: ainsi de quelques tentatives de calibrage de la loi de Reilly (par exemple, Moindrot, 1975) ou de divers modèles d'organisation régionale (Béguin, 1974); de l'application d'un modèle de diffusion à l'étude de l'implantation des établissements industriels dans le réseau urbain de l'Ouest français (SaintJulien, 1982); de l'adaptation du modèle d'A. Wilson aux navettes domicile-travail dans l'agglomération rouennaise (Guermond, 1982); de la vérification de plusieurs modèles concernant les rapports entre distribution des tailles de villes ("loi rang-taille») et répartition interurbaine de la croissance dans l'ensemble des villes françaises (Pumain, 1982).

Sans doute en raison de la rareté de ces applications et compte tenu de l'importance des débats épistémologiques contemporains évoqués ci-dessus, très peu de ces travaux ont été effectués dans l'optique stricte du test d'un modèle. Beaucoup d'auteurs ont cherché à expliciter les fondements de la démarche modélisatrice pour mieux l'insérer dans la pensée géographique française, ouvrant ainsi la voie à l'innovation. 
C'est sans doute la théorie des places centrales qui a suscité en matière de modélisation les recherches les plus clairement finalisées. On vient d'ailleurs de découvrir que cette théorie avait été inventée, cent ans avant Christaller, par un Français, Jean Reynaud (Robic, 1982). Les travaux d'H. Béguin ont démontré l'existence de compatibilités entre les hiérarchies urbaines prévues par la théorie et celles, observées, que résume la loi rang-taille (1979). J.-L. Piveteau (1968) a proposé une méthode d'analyse de la taille et de l'espacement des villes dans le réseau urbain suisse. J.-P. Martin (1977) s'est intéressé à la manière dont l'organisation hiérarchique d'un ensemble de villes assimile leur croissance démographique. Les recherches du Laboratoire de géographie théorique et quantitative de Strasbourg (1977) ont, au cours des dernières années, testé sur le réseau urbain alsacien différentes expressions des modèles issus de la théorie des lieux centraux. H. Reymond et C. Cauvin (1981) ont aussi exploré les potentialités de l'outil que constitue l'analyse spectrale pour l'étude des réseaux de villes. Ils ont montré l'acuité des problèmes de formalisation et d'évaluation des éléments du réseau - et donc de conceptualisation de celui-ci - avant toute utilisation efficace de la méthode d'analyse.

Dans le domaine de la modélisation proprement dite (c'est-à-dire de la fabrication de modèles) les recherches sont encore peu nombreuses, mais très prometteuses. Si les modèles urbains de la première génération avaient été relativement peu diffusés parmi les géographes, malgré des ouvrages comme celui de P. Merlin (1973), ceux qui s'inspirent actuellement de la dynamique des systèmes ont suscité beaucoup plus d'enthousiasme (Laurini, 1980).

La dynamique des systèmes permet de modéliser l'évolution des systèmes ouverts. Une ville, un réseau de villes, peuvent donc être désormais saisis comme des systèmes en interaction avec l'extérieur. Les hypothèses tout à fait irréalistes qui, dans les modèles statiques, réduisent les systèmes étudiés à des systèmes fermés peuvent donc être dépassées. De plus, en intégrant les chaînes causales d'interactions et de rétro-actions qui relient les éléments du système, la dynamique des systèmes apporte des solutions beaucoup plus satisfaisantes pour définir, à un moment donné, l'état du système et s'interroger sur son devenir. En définissant les concepts nécessaires pour une modélisation dynamique du système des transports québécois, H. Reymond (1974) a sensibilisé les géographes français aux exigences de cette démarche. Il a montré que plaquer un jargon systémiste sur n'importe quel discours géographique était un détour assez inutile qu'il fallait absolument dépasser.

En dynamique urbaine, les travaux de J. Forrester ont été de mieux en mieux connus (1979). Certains géographes français ont 
utilisé cet outil pour construire des modèles permettant de simuler le développement de villes françaises ou de régions. Les travaux de C. Alexandre (1976) sur Decazeville, ceux de C. Laudet et J. Fournier (1979) sur Carpentras sont une application assez directe du modèle de Forrester au cas de ces deux villes françaises. Les travaux du groupe AMORAL (1982) vont plus loin dans la réflexion et dans l'adaptation de l'outil pour une simulation du développement régional dans les Alpes du Sud.

Tous ces auteurs se sont toutefois heurtés à l'insuffisante prise en compte de la dimension spatiale dans cette famille de modèles. Aussi, des travaux d'application et d'élaboration de modèles urbains qui soient à la fois dynamiques et spatiaux sont en cours. Un modèle comme celui de P. Allen (1978), fondé sur l'analogie des structures dissipatives en physique, permet de simuler le développement d'une ville en tenant compte des interactions non linéaires (avec effets d'amplification ou de saturation), spatiales ou non spatiales, qui commandent la redistribution des emplois et des populations entre les différents quartiers. Il permet de prévoir diverses configurations possibles dans le futur à partir d'une histoire donnée. Ce modèle a été appliqué à la simulation du développement de l'agglomération de Rouen (Ozan et al., 1983).

Les études faites à partir de modèles dynamiques par simulation permettent seules de se rapprocher d'une méthode expérimentale, en précisant les effets de modifications de comportements, des règles du jeu de l'évolution du système, en faisant la part des "hasards locaux» et des contraintes déterministes d'ensemble. Ces études permettent de décrire des conséquences assez souvent contre-intuitives de telle ou telle intervention sur le système. Cependant, la tâche reste lourde car, en dépit des performances des outils proposés, le travail de conceptualisation que nécessite toute entreprise de modélisation fait prendre l'exacte mesure des carences théoriques et de la fragilité de certaines de nos connaissances empiriques par rapport aux interrogations formulées sur les villes.

Nous nous sommes livrés à une revue très concrète du rôle de l'outil informatique dans le développement récent des recherches urbaines. Pour aller plus loin dans l'imagination de ce que cet outil pourra apporter à ce champ de notre discipline, nous ne pouvons que conseiller au lecteur de méditer le texte d'H. Reymond (1981) sur L'Ouverture informatique en géographie urbaine. 


\section{Bibliographie}

Amoral, 1982, "Un modèle de dynamique de l'emploi dans un système régionalurbain", Communication au stage de géographie quantitative de Rouen (à paraître).

Andan O., Boislaroussie J.-J., Geiger P., 1976, "Types d'organisation des aires de relations des grandes villes françaises ", Analyse de l'Espace, n², 107 p.

Andan O., 1982, "Mobilité et espace urbain. Etude longitudinale des comportements de mobilité en fonction d'un changement de résidence", Rapport au ministère des Transports, Mission de la Recherche.

Auriac F., Bruynooche M., 1978, "Une classification sur plus de 1500 communes: la distance à la ville en Languedoc-Roussillon ", Cahiers de Géographie de Besançon, pp. 107-217.

Bailly A., 1975, L'Organisation urbaine: modeles et theories, Paris, Centre de Recherche d'Urbanisme, $272 \mathrm{p}$.

Bailly A., 1977, La Perception de l'espace urbain. Paris, Centre de Recherche d'Urbanisme.

Bailly A., Polèse M., 1976, "Analyse typologique en milieu urbain: les aires homogènes d'Edmonton ", L'Espace géographique, n 4, pp. 267-275.

Bailly A., Polèse M., 1978, "La différenciation de l'espace urbain de Québec ", Cahiers de Géographie du Québec, n'22, pp. 29-49.

Barthélemy J.-P., Luong X., 1978, "Sur l'attraction des villes et des villages d'une région", Cahiers de Géographie de Besançon, pp. 179-192.

Beaujeu-Garnier J., sous la direction de, 1967, Atlas de Paris et de la région parisienne, Paris, Berger-Levrault.

Beaujeu-Garnier J., 1971, La Géographie: méthodes et perspectives, Paris, Masson, $142 \mathrm{p}$.

Béguin H., 1974, L'Organisation de l'espace au Maroc, Bruxelles, Académie royale des Sciences d'Outre-Mer, $787 \mathrm{p}$.

Béguin H., 1979, "Urban Hierarchy and the Rank-Size Distribution", Geographical Analysis, vol. 11, n"2, pp. 149-163.

Berry B.J.L., 1964, "Cities as Systems within Systems of Cities", Economic Development and Cultural Change, 9, pp.573-587.

Berry B.J.L., 1967, Geography of Market Centers and Retail Distribution, EnglewoodCliffs (N.J.), Prentice Hall, 146 p.

Berry B.J.L., 1972, "Latent structure of the american urban system with international comparisons", in Berry B.J.L. (ed.), City Classification Handbook, New York, J. Wiley, pp. 11-60.

Bouinot J., Maarek G., 1974, "Les grandes agglomérations et leurs finances", Economie et Statistique, n" 58, pp. 15-32.

Brun J., 1981, "Ecologie urbaine et géographie sociale", Villes en parallele, n 4, 31 p.

Carrière F., Pinchemel Ph., 1963, Le Fait urbain en France, Paris, A. Colin, 374 p.

Castells M., 1972, La Question urbaine, Paris, Maspero, 454 p.

Castells M., Godard M., 1974, Monopolville, analyse des rapports entre l'Entreprise, l'Etat et l'Urbain, Paris, Mouton, $435 \mathrm{p}$.

Cauvin C., 1981, La Perception des distances en milieu intra-urbain, tome 1: Techniques de collecte; tome 2: Distance chorotaxique et distance cognitive, Strasbourg, $80 \mathrm{p}$. et $100 \mathrm{p}$. dactylographiées.

Cauvin C., Reymond H., 1980, Cartographie informatisée et géographie humaine. C.N.R.S., A.T.P., Expérimentations en cartographie transformationnelle, tome 2, $150 \mathrm{p}$.

Chabot G., 1961, Carte des zones d'influence des grandes villes françaises, Mémoires et Documents du C.R.D.C.G., tome 8, Paris, C.N.R.S., pp. 139-143.

Chatelain A., 1956, "Géographie sociale des villes françaises en 1946 ", Revue de Géographie de Lyon, 2, pp. 119-127. 
Chatelain A., 1981, "Analyse des morphologies urbaines et de leur évolution à partir des données de télédétection". Informatique et Sciences humaines, n 50, pp. 83-105. Chauviré Y., Noin D., 1980, "Typologie socio-professionnelle de l'agglomération parisienne", B.A.G.F., pp. 467-470.

Claval P., 1968, "La théorie des villes", Revue géographique de l'Est, n 1-2, pp. 3-56.

Claval P., 1981, La Logique des villes, Paris, Litec, 633 p.

C.N.R.S., 1982, Actes des Journées Télédétection en milieu urbain, 6-7 mai, Paris, C.E.R.C.G., 140 p.

Dalmasso E., Cauvin C., Pruvot M., Rimbert S., 1971, "Essai d'application de quelques méthodes statistiques à la région milanaise", Bulletin de l'Association des Géographes français, n’393, pp. 377-392.

Debonneuil X., Gollac M., 1978, "Structure sociale des villes", Economie et Statistique, n'98, pp. 51-65.

Dreyfus J., Chasseriaux E., 1972, "Applications de l'analyse factorielle à des problèmes urbains", Colloque sur l'analyse des données en géographie. Cahiers de Besançon, n० 8.

Dreyfus J., 1976, La Ville disciplinaire, Paris, Editions Galilée, 215 p.

Dupont, Groupe, 1975, "La distance à la ville, essais d'analyses factorielles appliquées aux cas de Grenoble et de Montpellier ", L'Espace géographique, n ${ }^{\oplus} 4$, pp. 225-238.

Dupont, Groupe (Montpellier), 1980, Montpellier 1954-1978: processus de transformation de l'espace et développement du bâti urbain, Montpellier-Caen ed., $238 \mathrm{p}$.

Durand-Dastès F., 1979, Aspects de la localisation des industries dans les agglomérations urbaines de l'Inde, Travaux de l'Institut de Géographie de Reims.

Fontanel G., Peseux C., 1976, "Potentiel de population et réseau urbain en France", $n^{\circ} 4$, pp. $251-254$.

Forrester J.-A., 1979, Dynamique urbaine, traduction française, Paris, Economica, $325 \mathrm{p}$.

Grafmeyer Y., Joseph, 1979, L'Ecole de Chicago, naissance de l'écologie urbaine, Paris, Ed. du Champ urbain.

Guermond Y., 1981, "Micro-informatique et analyse multivariée: la structure socio-proféssionnelle de l'agglomération de Rouen", Brouillons Dupont, n 07.

Guermond Y., Langlois P., Lannuzel B., Lascaux F., 1982, "The Gravity Model: Empirical Test and Improvments in a French City", Communication au colloque européen de Géographie théorique et quantitative, Augsburg, 13-17 sept.

Haumont A., Bohain C., 1968, "Quelques caractéristiques des agglomérations françaises de plus de 50000 habitants en $1962 \%$, Revue française de Sociologie, vol. IX, n², pp. 222-250.

Hautreux J., Lecourt R., Rochefort M., 1963, Le Niveau supérieur de l'armature urbaine française, Paris, ministère de la Construction, Commission de l'Equipement Urbain, $60 \mathrm{p}$.

Herin M., 1981, "Différenciations et ségrégations résidentielles dans la grande banlieue Ouest de Paris", Revue d'Economie régionale et urbaine, n 1, pp. 3-32.

Holz. J.-M., 1974, "Essai de classification des villes de la Ruhr", Revue géographique de l'Est, n'3-4, pp. 313-330.

Laboratoire de Géographie Théorique et Quantitative de Strasbourg, 1977, "Analogie, homologie, explication en géographie humaine», Recherches géographiques à Strasbourg, $\mathrm{n}^{\circ} 2,141 \mathrm{p}$.

Lacour C., 1970, "Quelques récents résultats de recherches en matière d'armature urbaine ", Metra, 1(9), pp. 13-56.

Laudet C., Fournier S., 1979, "La simulation de politiques urbaines. Essai d'application du modèle de Forrester à une ville moyenne", Rapport de recherche D.A.T.A.R., 123 p.

Lebart L., Tabard N., 1971, "La morphologie sociale des communes urbaines", Consommation, $\mathrm{n}^{\circ} 2$, pp. 97-107. 
Ledrut R., 1968, L'Espace social de la ville, Paris, Anthropos, $370 \mathrm{p}$.

Lefebvre H., 1968, Le Droit à la ville, Paris, Anthropos, $164 \mathrm{p}$.

Lipietz A., 1977, Le Tribut foncier urbain, Paris, Maspero, $290 \mathrm{p}$

Lowy P., 1975, "Les villes fermées d'Afrique du Nord, méthodes de recherche", L'Espace géographique, $\mathrm{n}^{\circ} 1, \mathrm{pp} .31-43$.

Marchand B., 1978, La Croissance de Los Angeles, Paris, Thèse d'Etat, 463 p.

Martin J.-P., 1977, «La croissance sélective des lieux centraux de la province du Québec ", Recherches géographiques à Strasbourg, n 6, pp. 13-49.

Merlin P., 1973, Méthodes quantitatives et espace urbain, Paris, Masson, $190 \mathrm{p}$.

Meunier M., Racine J.-B., 1977, «Richesse et limites de l'induction quantitative en géographie: l'exemple de la réduction factorielle des attributs de l'agglomération lausannoise ", Cahiers de Géographie de Besançon, pp. 114-160.

Moindrot $\mathrm{Cl}$., 1975, "La délimitation des aires d'influence métropolitaine par un modèle de gravité : le Centre-Ouest de la France ", L'Espace géographique, n" 3, pp. 197-208.

Ozan A., Pumain D., Saint-Julien Th., Sanders L., 1983, "Application d'un modèle de dynamique intra-urbaine à l'agglomération rouennaise ", Cahiers de Géographie de Rouen (à paraître).

Pierchon M., 1979, "Une typologie des quartiers urbains en Languedoc-Roussillon ", Société languedocienne de Géographie, $\mathrm{n}^{\circ} 1,33 \mathrm{p}$.

Piolle X., 1979, Les Citadins et leur ville, Ed. Privat, $423 \mathrm{p}$.

Piveteau J.-L., 1968, "Le "rang de voisinage" comme moyen d'approche de l'influence urbaine et de l'environnement urbain. Application à la région comprise entre Berne et Lausanne", Revue de Géographie de Lyon, n 4, pp. 377-394.

Pumain D., 1976, "La composition socio-professionnelle des villes françaises. Essai de typologie par analyse des correspondances et classification automatique ", L'Espace géographique, $\mathrm{n}^{\circ} 4$, pp. 227-238.

Pumain D., 1981, "Peut-on modéliser la répartition de la croissance urbaine?" Informatique et Sciences humaines, $\mathrm{n}^{0}$ 50, pp. 61-82.

Pumain D., 1982, La Dynamique des villes, Paris, Ed. Economica, 231 p.

Pumain D., 1980, Contribution à l'étude de la croissance urbaine dans le système urbain français, université de Paris I, Thèse de Doctorat d'Etat, $492 \mathrm{p}$.

Pumain D., Saint-Julien Th., 1978, Les Dimensions du changement urbain, Paris, Ed. du C.N.R.S., 202 p.

Pumain D., Saint-Julien Th., 1979, "Les transformations récentes du système urbain français", L'Espace géographique, n 3, pp. 203-211.

Pruvot M., Kleinweber Ch., 1980, "Pour une théorie factorielle en géographie ", Actes du Colloque Géopoint, $4 \mathrm{p}$.

Racine J.-B., 1971, "Le modèle urbain américain", Annales de Géographie, n440, pp. 397-427.

Racine J.-B., 1973, Un Type nord-américain d'expansion métropolitaine: la commune urbaine du grand Montréal. Géographie factorielle expérimentale d'un phénomene suburbain, Thèse de Doctorat d'Etat, Nice. Service de reproduction des thèses, Lille, 1975.

Racine J.-B., 1973, «La centralité commerciale relative des municipalités du système métropolitain montréalais ", L'Espace géographique, n' 4, pp. 275-289.

Reymond H., 1974, Analyse géographique d'une modélisation gravitaire, Nice, Thèse d'Etat, $376 \mathrm{p}$.

Reymond H., 1981, "L'ouverture informatique en géographie urbaine: de l'analyse multivariée socio-économique à la simulation organique des systèmes urbains", Informatique et Sciences humaines, $\mathrm{n}^{\circ}$ 50, pp. 9-20.

Robic M.-C., 1982, "Cent ans avant Christaller... une théorie des lieux centraux", L'Espace géographique, tome XI, $\mathrm{n}^{\circ} 1$, pp. 5-12. 
Rolland-May Ch., 1978, "L'urbanisation du pays messin», L'Espace géographique, $n^{\circ} 1$, pp. 43-51.

Roncayolo M., 1972, "La division sociale de l'espace urbain", Bulletin de l'Association des Géographes français, $\mathrm{n}^{\circ} 395$, pp. 3-20.

Saint-Julien Th., 1982, Croissance industrielle et systeme urbain, Paris, Economica, $275 \mathrm{p}$

Saint-Julien Th., 1981, "Techniques statistiques et analyse d'un processus d'industrialisation ", Informatique et Sciences humaines, n० 50, pp.41-60.

Vant A., 1981, Imagerie et urbanisation; recherches sur l'exemple stéphanois, Centre d'Etudes foréziennes, $627 \mathrm{p}$.

Vigouroux M., 1980, "Bricolage : un modèle réduit d'écologie factorielle", Brouillons Dupont, $\mathrm{n}^{\circ} 6$.

Vigouroux M., 1981, "Les données du milieu urbain: formalisation et préliminaires aux traitements informatiques ", Informatique et Sciences humaines, $n^{\circ} 50$, pp. 21 40.

Vigouroux M., Volle J.-P., 1982, "L'espace social des centres et quartiers traditionnels à Nîmes, Montpellier et Perpignan: un essai d'écologie factorielle ", Bulletin de la Société Languedocienne de Géographie, juillet-décembre, pp. 287-320.

Résumé. - Comment et pourquoi l'analyse quantitative s'est progressivement imposée auprès d'une minorité importante de chercheurs français en géographie urbaine? L'article répond à ces questions. L'incursion des géographes urbains dans ces nouveaux champs méthodologiques et techniques a beaucoup contribué à la vitalité des recherches urbaines et ce pour plusieurs raisons. La première est que grâce à la généralisation de l'outil informatique et aux possibilités de l'analyse multivariée des vérifications jusque là irréalisables ont pu être faites dans différents domaines, et que par ailleurs de nouveaux champs d'observation ont été ouverts (exemple: la télédétection). La seconde est que les démarches mises ainsi en cuvve ont renvoyé le chercheur à des exigences beaucoup plus grandes en matière d'explicitation théorique, de conceptualisation, et de rigueur de l'observation. Les tenants de cette démarche se trouvent donc très étroitement impliqués dans l'ensemble des débats et confrontations qui animent depuis une dizaine d'années la géographie urbaine française. Aujourd'hui, les voies ouvertes par la modélisation et l'analyse des systèmes apportent de nouvelles perspectives à l'investigation urbaine.

Abstract. - How and why has quantitative analysis imposed itself upon an important minority of French urban geographers? This article answers these questions. The urban geographers'entry into these new methodological fields and these new techniques has much contributed to the vitality of urban studies for several reasons. First the spreading of computers and the possibilities of multivariate analysis have enabled to make verifications so far impossible to perform in several fields; besides new means of study have opened up (e.g. remote sensing). Second such processes are far more demanding regarding the theoretical explanation, the conceptualization and the precision of observation of the phenomena. The followers of these processes are thus very much involved in the debates that have been at the center of French urban geography for the last ten years or so. To-day model-making and system-analysis are opening new ways and hopes for the urban studies. 